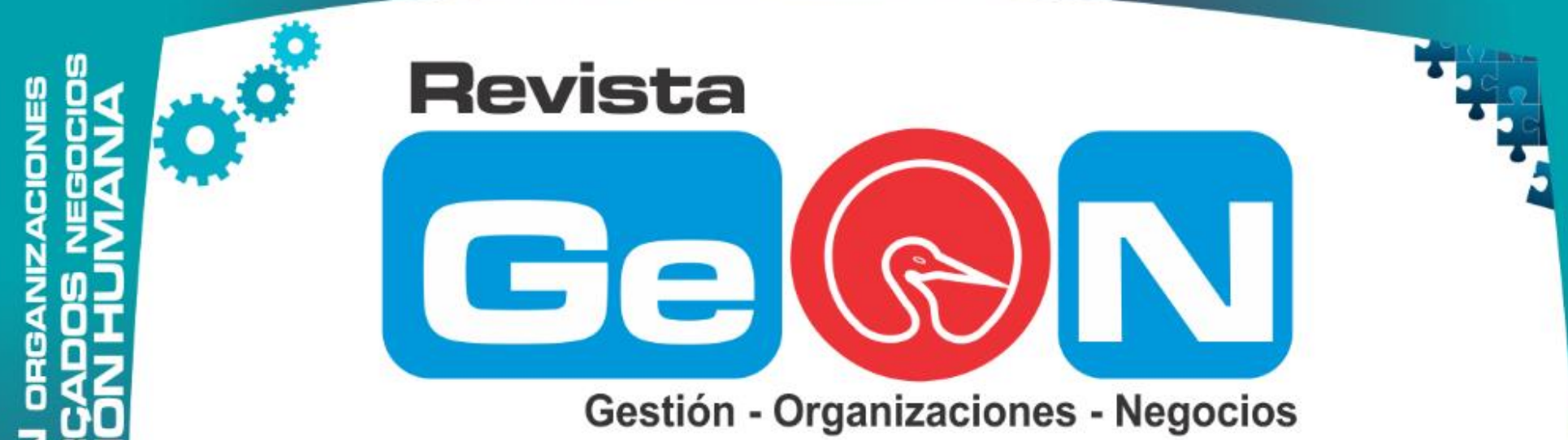

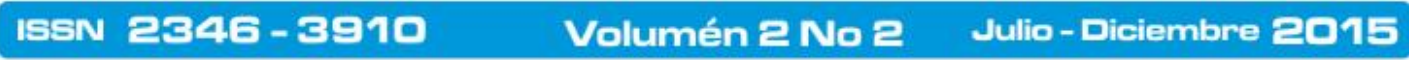

Revista Electrónica de la Facultad de Ciencias Económicas de la Universidad de los Llanos

1. 3 II

440

VILLAVICENCIO - COLOMBIA

$>2$

64

2

2 平品

70

ব)

展

o

ii III

0 U

ใด

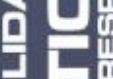

居的

임

Iiin in

照

只

造

iil 2

if in

4 吕

2

400

付 iI
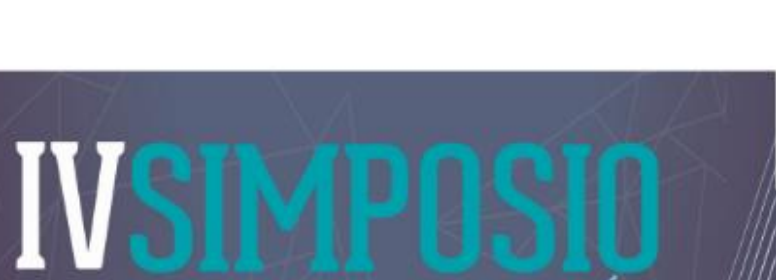

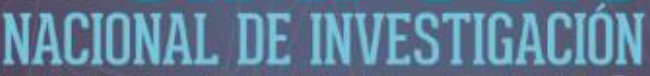

EN MARKETING
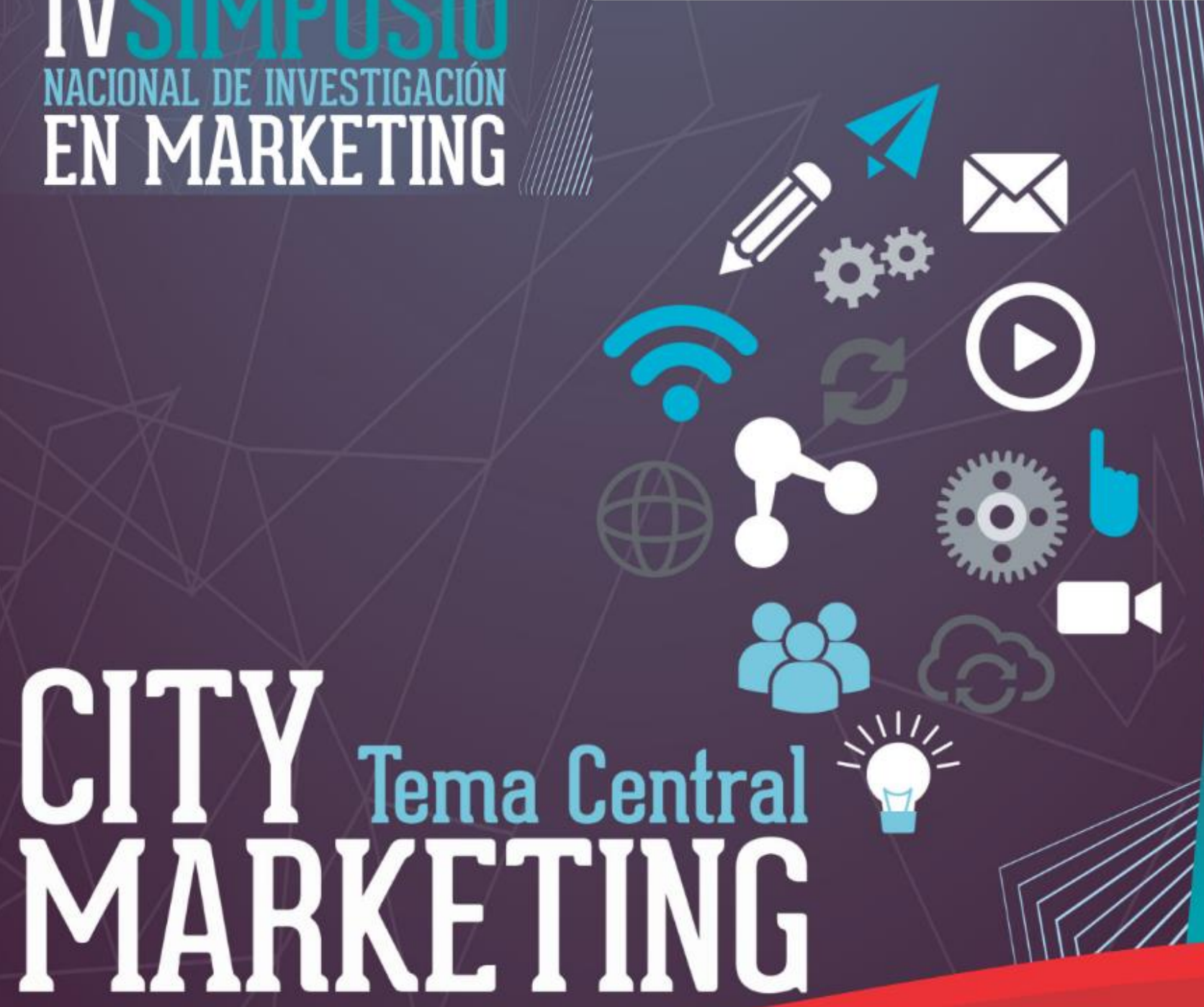

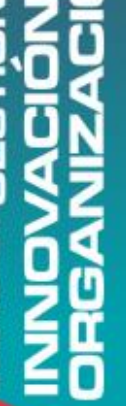
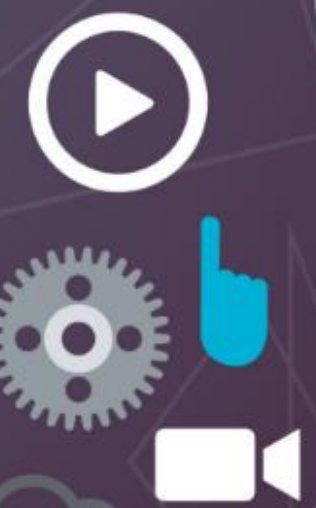


\section{Manejo empírico y emergente del espacio interno de ventas de tiendas de barrio en Bogotá - Luis Marquez}

Luis Fernando Márquez Valencia

Maestría en Ciencias Económicas y

Administrativas

lmarquez@ucentral.edu.co

\section{Resumen}

El presente artículo buscó conceptualizar desde el pensamiento complejo con observaciones y diálogos cómo se maneja la exhibición y el espacio interno de ventas de un grupo de tiendas de barrio de la Localidad de Barrios Unidos en la ciudad de Bogotá. Partió de los conocimientos y experiencia del autor y del diálogo individual con un grupo de tenderos de la citada Localidad. A través de su propio lenguaje y sus interacciones, para comprender como manejan el objeto descrito, generando una nueva mirada sobre el asunto. Para ello se usaron métodos cualitativos de investigación y recolección de información, como la observación y las entrevistas de profundidad de donde emergieron varias percepciones, que permiten entender la forma como los tenderos han manejado la exhibición y los espacios internos de ventas de sus tiendas.

\section{Palabras Clave}

Tiendas barrio, merchandising empírico, emergente

\begin{abstract}
This article sought to conceptualize from the complex thought with comments and dialogues how the internal space exhibition and sales of a group of neighborhood shops of the town of Barrios Unidos in Bogota is handled. He left the knowledge and experience of the author and individual dialogue with a group of shopkeepers in the same town. Through its own language and their interactions, to understand how they handle
\end{abstract}

the object described, creating a new look at the issue. For this qualitative research methods and data collection, and the observation and depth interviews where several perceptions emerged, for understanding how the shopkeepers have handled the display and the internal spaces of store sales were used.

\section{Keywords}

Stores neighborhood, empirical and emergent merchandising,

\section{Introducción}

La tienda de barrio, como fenómeno socioeconómico y comercial se construye con la interacción de los deseos de superación y emprendimiento de sus propietarios, los tenderos, por medio de las necesidades de compra y consumo de sus vecinos, los esfuerzos de distribución y venta de sus proveedores productores y comercializadores, entre otros aspectos.

A pesar de ellos, no siempre ha sido suficientemente entendido el papel que juega el tendero de barrio en la sociedad, no solamente como impulsor de acciones sino en la construcción misma de un tipo diferente de actividad comercial, así como del vínculo mercadológico y socio-cultural con la comunidad a la cual atiende. Esto se debe a que la tienda de barrio generalmente ha sido vista como un proveedor de artículos de la canasta familiar o un instrumento para satisfacer las necesidades primarias, antes que un canal de distribución especial por sus características, dentro de los mercados de productos de consumo masivo en Latinoamérica y Colombia, creando un sistema de vínculos con características propias. En tanto que este canal de distribución comienza a evolucionar como un sistema de 
acuerdos y contratos verbales que coordinan conductas, plasmadas en el famoso "fiado", o venta de productos a crédito y en las relaciones humanas, se percibe su carácter dialógico (Flores, 1994).

Luego de efectuar estas precisiones, se consideró útil recurrir al pensamiento complejo, a marcos teóricos diferentes a aquellos que tienen una visión tradicional, que develan perspectivas causa-efecto, en donde los tenderos, no tienen cabida en el proceso de construcción de la estructura comercial deliberada, sino en manifestaciones comerciales espontaneas, sin ningún tipo de planificación.

Basados en la gran riqueza de las conversaciones en este estudio, se trató la forma de lograr información para comprender como han logrado subsistir ante el fuerte ataque de las grandes cadenas detallistas de productos de consumo masivo, con una herramienta mercadológica, como el "merchandising

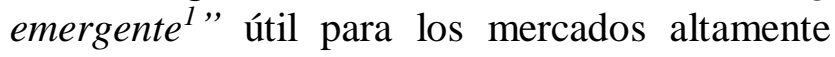
competidos, como en el que se encuentra las tiendas, quienes no sólo compiten entre sí, sino con los otros miembros de los sistemas de distribución minorista masiva, como los autoservicios, hipermercados y otros detallistas como las droguerías, panaderías, carnicerías, tiendas de frutas y verduras o "líchigo", como las llaman de manera popular, etc.

En estas tiendas de barrio, que sin ningún tipo de orientación, capacitación, planeación, etc. realizan su propia "auto organización ${ }^{2}$ " con un alto grado de informalidad, no se percibe la

1 Se entiende por aquellas exhibiciones, manejo de espacios internos de ventas, acciones realizadas por los tenderos de manera espontánea, etc., fruto de su interrelación con el mercado y sus diferentes agentes.

2 De acuerdo con los estudios de John Von Neumann, padre del modelo de arquitectura de la computadora, en su teoría de las maquinas, se plantea la diferencia entre las maquinas artificiales y las maquinas vivas, es decir los humanos, quienes como maquinas tiene la capacidad de desarrollarse, reproducirse, autogenerarse reemplazando las moléculas deterioradas. existencia del merchandising, como se promulga en las empresas de consumo masivo, universidades y demás centros técnicos, su importancia es relativa a cada tendero.

Tampoco se ha manejado de una manera estructurada y sostenible por los mismos, sino por los diferentes proveedores de productos de consumo masivo, a quienes en algunas ocasiones se les facilitan los espacios para que lo ejerzan, ya que la mayoría de los tenderos entrevistados, ignoran muchas de las estrategias y tácticas sugeridas por las empresas proveedoras, lo cual se traduce en resultados negativos, para dichas entidades.

Esto ha llevado a que con base en sus necesidades y expectativas, las fortalezas y las debilidades de sus propios establecimientos, a sus experiencias y conocimientos empíricos, entre otras variables los tenderos construyan, lo ya citado y denominado en esta investigación, como manejo "emergente" de exhibición y espacio de ventas de tiendas de barrio, conocido como "merchandising emergente 3 "'En lo relacionado con el estudio de los antecedentes se hizo una búsqueda relacionada con el objeto de investigación, si bien se encontraron muchos documentos y artículos de producción intelectual, por ser este tema de las tiendas de barrio ampliamente tratado con fines mediáticos, tales como estudios cuantitativos de mercadeo, estudios económicos, psicológicos, sociológicos, antropológicos, etnográficos, etc. no se encontraron relacionados con el "merchandising empírico y emergente”, lo cual nos indica lo novedoso de este estudio.

Así entonces, este documento expone brevemente una caracterización de las tiendas de barrio, el origen del problema, los métodos utilizados, el énfasis dado al paradigma de la complejidad, la investigación cualitativa, etc., para construir un marco teórico. Luego se presentan los resultados, la discusión, el sumario y las referencias bibliográficas. 


\section{Desarrollo}

\section{El merchandising y NO merchandising de los tenderos}

Merchandising, etimológicamente viene de la palabra en inglés, "merchandise", que significa mercancía o producto. La terminación en "ing", en inglés significa acción de movimiento hacía, lo cual nos da a entender que el movimiento hacía, es mover o empujar el producto hacía el comprador. Que es un sinónimo de la promoción o "estrategias push"”, (Kotler, 2009).

Según Díaz de Castro, (2006), el merchandising son las acciones de mercadeo realizadas en el punto de venta tipo autoservicio, con el fin de generar ventas de los productos allí exhibidos y ofrecidos a los compradores $\mathrm{y}$ clientes, buscando satisfacer sus necesidades compra y consumo, como las de rentabilidad del espacio dedicado por parte del dueño del establecimiento comercial. Ante todo es el manejo adecuado de los estantes (espacios), en el surtido (variedad de productos ofrecidos en amplitud y profundidad), la presentación de los productos, el circuito realizado por los clientes en el proceso de compra, la animación entendiendo por esta la forma de destacar el producto en el almacén, la gestión y rotación de la mercancía en el lugar, la cual se basa en la entrada y salida de inventario de productos, los espacios donde se ubican los productos de altos niveles de ventas y rotación se denominan "calientes", como los de bajos "fríos", el llamado material p.o.p, por sus siglas en inglés, "point of purchase", en español punto de compra, o material de propaganda entre otros. Al contrastar la teoría anterior con lo encontrado en la investigación, vemos que, podemos confirmar lo afirmado anteriormente, en el sentido de que no podemos aseverar que los tenderos manejen "merchandising" como tal, ya que su sistema de venta no es el de un autoservicio, sino ventas de

\footnotetext{
${ }^{4}$ Es igual en español a estrategias de empuje de un producto en un canal de distribución
}

mostrador, por medio de un tendero o un vendedor dependiente.

En muchas tiendas de barrio, no se sabe la rentabilidad, ya que no se lleva ningún tipo de contabilidad. Los estantes de los establecimientos de los tenderos presentan un manejo sin ningún tipo de lineamiento técnico, puesto que exhiben los productos como pueden en los pequeños espacios disponibles.

El surtido no pretende prestar un servicio al cliente, ya que como los tenderos manifestaron, "lo que no se vende estorba", en el sentido de que si un comprador desea un producto que se vende muy poco, no lo encontraran en la tienda de barrio, ni por amplitud, ni por profundidad. En las tiendas de barrio no se pueden hacer circuitos, ya que no hay espacio para serlo, como en el merchadising tradicional, además de que los productos no se exhiben como en los autoservicios, quienes generalmente exhiben en góndolas, para que los compradores los puedan tomar directamente. Generalmente destacan los productos que están próximos a vencerse, sin ningún tipo de estrategia o táctica visible. Los inventarios los manejan por sentido común, los primeros en entrar, son los primeros en salir, pero no desde el punto de vista contable y de costos.

En cuanto al manejo de los espacios no existe un lineamiento general, algunos ponen los productos que más se vende en los espacios "calientes", otros tenderos ponen los que menos se venden y al contrario con los exhibidos en los espacios fríos. En lo relacionado con el material p.o.p, en el cual las empresas proveedoras invierten ingentes sumas de dinero, para muchos tenderos es contaminación visual que deteriora las paredes y resta belleza a sus tiendas, el cual está proscrito, ya que tampoco les ayuda a incrementar las ventas, según lo expresado por ellos, incluso algunos tenderos hacen su propio material de manera "artesanal", para promover o anunciar los productos que les interesan.

Varios tenderos manifestaron cómo ellos manejan su tienda de manera autónoma, sin 
permitir que ningún proveedor intervenga en la organización y distribución de la tienda.

\section{Características de las tiendas de barrio involucradas en el estudio}

Antes de precisar las características tratadas para desarrollar la investigación como "vehículo metodológico", debo aclarar que el factor determinante en este estudio en lo relacionado con el tipo de tiendas de barrio, sobre las cuales se hizo esta investigación, como se dijo anteriormente es la heterogeneidad, en otras palabras, por aspectos metodológicos de determinar un perfil de estudio, o lo que en mercadología llamamos un segmento objetivo de estudio, usamos los factores que a continuación enuncio, pero la realidad encontrada fue bien diferente, ya que no podemos hablar de uniformidad $\mathrm{u}$ homogeneidad, puesto que este fenómeno desde ningún punto de vista se puede estandarizar y es una de la justificaciones ontológicas y epistemológicas de porque se trata con lineamientos del paradigma de la complejidad y pensamiento complejo, no del pensamiento lineal tradicional, del paradigma de la simplicidad, orientado por relaciones positivistas causa - efecto.

Las características de las tiendas de barrio fueron: a) Área en metros cuadrados 50 b) No exhiben en forma de autoservicio c) Las ventas son de mostrador d) Tiene vendedores dependientes que atienden la tienda e) $\mathrm{Su}$ organización es familiar o famiempresa f) Una caja registradora g) Tipo de productos vendidos frutas y verduras, carnes, lácteos, abarrotes (alimentos y no alimentos), licores, bebidas, cigarrillos, productos de panadería, etc.

\section{Sistemas humanos, conversaciones, complejidad}

Una posibilidad para adquirir nuevas comprensiones acerca de cómo interactuar los sistemas humanos, en particular los tenderos de barrio que atraviesan por dificultades asociadas al ejercicio de sus objetivos funcionales, la ofrece la visión compleja (Morín, 1997). Esta visión ha ubicado a la organización como parte esencial del trabajo con los sistemas humanos, al complejizar los procedimientos para la investigación empresarial de manera integral. Esta perspectiva ofrece marcos que invitan a la participación de más contextos y de mayores posibilidades de interconexión de conversaciones y relaciones (Anderson, 1977).

En la misma dirección, desde el punto de vista de estos, los sistemas humanos, estudiosos de las conversaciones reflexivas, de los lenguajes y modos de proceder que habitan estas conversaciones como Gergen (2007), Markova (2000), basados en planteamientos de Wittgenstein (1950), Bateson (1972), Maturana (1996), Foucault (1969), aportan al estudio del lenguaje un efecto catalizador de las relaciones que se dan con las personas dentro de una realidad social, vistas como sistema, que mediante una orientación particular en ese intercambio es posible alcanzar un significado, que lleva a una comprensión particularizada, desde la cual emergen innovaciones comerciales.

Desde una visión compleja, se considera que los tenderos pueden aportar soluciones importantes desde su experiencia y vivencia, ya que por estar inmersos en los problemas, pueden aportar en la deconstrucción o solución de los mismos. En el caso que nos ocupa en el manejo de sus espacios de las áreas internas de ventas, el material de propaganda (material pop ${ }^{5}$ ) y demás actividades comerciales que han desarrollado físicamente por puro instinto, en este caso lo que mercadológicamente se llama "merchandising".

Por otro lado, resulta útil considerar las fortalezas que tiene una tienda de barrio vista como sistema capaz de auto organizarse $y$ sostenerse en mercados altamente competitivos, según lo planteado en 1971 por Heinz Von Foerster. Es decir, aquella capacidad sistémica que aplicada a las empresas le permite operar, conservar los elementos y articulaciones que requieren, para funcionar sin perder sus

\footnotetext{
${ }^{5}$ Del inglés point of purchase, (punto de compra).
} 
características que la hacen un canal de distribución de productos de consumo masivo al detal único y muy particular, adaptado por los tenderos a las realidades del mercado del barrio, basado en su experiencia y conocimientos empíricos, que le permiten tener una identidad propia, diferenciándola así como una entidad única entre las demás (Etkin y Schvarstein, 1995).

Los instrumentos empíricos que también aportan a la búsqueda de una lógica del orden y la racionalidad dentro de esa identidad, juegan un papel primordial, como motores generadores de los ingresos de la tienda de barrio dentro de los funcionamientos de las estrategias emergentes de ventas, de manejo de sus exhibiciones y de sus espacios internos de ventas. En esta perspectiva, los citados instrumentos desempeñan un papel de homogenización o alineación dentro de las dualidades del orden y del desorden, las fortalezas y las debilidades, de acuerdo con las unas metas que si bien no se establecen de manera arbitratia y planeada, están implícitas en el proceder del tendero. Por lo que a un mercadólogo tradicional al tratar de precisarlas, desde un punto de vista simplista y lineal, le serían inexistentes en las tiendas de barrio.

Que no manejan conceptos globales de ventas de la empresa. Contrario al caso del positivismo y funcionalismo, de los canales de distribución formales y legalmente constituidos, en donde no se aceptan ningún tipo de ambigüedades. En otras palabras, dentro de la vida real de los hipermercados, cadenas de supermercados, tiendas por departamento, superetes, etc., cumplió con su cuota de ventas o no lo hizo. Aquí no cuentan las razones de la complejidad o incertidumbre. Que antagónicamente si le aplica a las tiendas de barrio, donde en gran mayoría, no llevan contabilidad, ni manejan prepuestos de ventas, compras, etc.

El pensamiento complejo y las tiendas de barrio: Desde el pensamiento complejo decimos que para comprender la realidad organizacional de las tiendas de barrio, se requiere incorporar el concepto de la incertidumbre de la información, relaciones dialógicas, sistémicas, holográmaticas, cibernéticas, autopoieticas, recursivas, etc. González, (2008). Esto indica que, en las tiendas de barrio, sus componentes están en relación compleja, que es a la vez complementaria, antagónica y concurrente.

Para constatar las acepciones complejas manifestadas haré una breve aplicación de ellas:

Dentro del contexto pedagógico definiré el concepto, para luego proceder a aplicarlo a la investigación efectuada:

La información en la complejidad "está caracterizada por la falta de certeza, la sorpresa y lo inesperado", (González, 2008) pp.16. En el caso que nos ocupa, las tiendas de barrio no tiene nada seguro, como pueden llegar a realizar muy buenas ventas, pueden tener que cerrar, como pueden tener dinero para pagar los productos a exhibir, pueden que pedirle al proveedor que vuelva otro día.

Las relaciones dialógicas, el diálogo une acciones "antagonistas, que aparentemente debían rechazarse entre sí, pero que son indisolubles para comprender una misma realidad" (González, 2008) pp.20. En el objeto investigado, las relaciones comerciales y en los diálogos que se establecen con algunos de los proveedores de las tiendas de barrio, buscan según palabras de los mismos tenderos "imponer" sus formas de exhibir y manejar los espacios internos de ventas, lo cual no es aceptado por muchos tenderos, no obstante, el proveedor termina aceptando las condiciones del tendero y también este accede a algunas de las disposiciones del proveedor, por ejemplo darle un espacio adicional a cambio de una oferta.

Las relaciones sistémicas, "el todo es más que la suma de las partes" (González, 2008) pp.17. Muchos tenderos manifestaron que para ello lo más importante es su tienda, independientemente de los múltiples productos y categorías de productos que vendan o dejen de vender en ellas. 
Las relaciones hologramáticas, "no solamente la parte está en el todo, sino que el todo está en la parte" (González, 2008) pp.21. La tienda está dentro del canal de distribución, el cual hace el $57 \%$ de las ventas de productos de consumo masivo en Colombia, según Fenaltiendas. Este canal es manejado por los fabricantes y proveedores como un todo, con una estrategia de ventas y distribución propia para el canal, que se refleja en cada una de las tiendas visitadas, que si bien no son muy exitosos, es una realidad que se presenta.

Las relaciones cibernéticas, "Bucle de retroacción, llamado feed-back” (González, 2008) pp.17. Si un cliente y comprador de una tienda de barrio, es cumplido y seguro en sus pagos al tendero, se "ganará" con el tendero el beneficio del "fiado", es decir de compras a crédito. Otro ejemplo si el tendero le da un espacio adicional en la tienda al proveedor y el producto rota o se vende adecuadamente, el tendero lo mantendrá en este espacio adicional al que tenía el proveedor y de esta forma este se retroalimentará con buenas ventas.

Las relaciones autopoiéticas, "la característica más peculiar de un sistema autopoiético es que se levanta por sus propios cordones y se constituye como distinto del medio circundante a través de su propia dinámica, de tal manera que ambas cosas son inseparables" Maturana, citado por Torres Nafarrete (1995) a su vez citado por González, (2008) pp.51, significa que los seres humanos se auto organizan y reproducen continuamente, este fenómeno se produce permanentemente en las tiendas de barrio, cuando un tendero exhibe un producto que le genera pérdidas por bajas rotación, inmediatamente toma la decisión de exhibirlo en otro lugar o dejarlo de comprar y cambiarlo por otro proveedor que le permita recuperar el dinero sin perderlo, volviendo a construir la relación simbiótica, venta de producto - exhibición del producto.

Las relaciones de recursividad, "Es un bucle generador en el cual los productos y los efectos son ellos mismos productores y causadores de lo que los produce" En cuanto a los tenderos de barrio ellos construyen sus propias exhibiciones de acuerdo a su sentido común y se las ingenian con su experiencia, con su mecanismo empírico de prueba y error, etc. si esta exhibición genera buenas ventas y relaciones positivas con sus clientes, ellos mismos le cuentas a los vendedores de los proveedores de sus formas exitosas de exhibición los cuales serán replicados por estos vendedores en otras tiendas, permitiendo regenerar no solo sus propias exhibiciones y mejorar sus ventas, sino las de sus colegas y las de la empresa proveedora en otras tiendas.

\section{Conclusiones}

Los hallazgos más representativos de la investigación son:

Las prácticas de merchandising de los tenderos de la Localidad de Barrios Unidos, no reflejan el merchandising convencional, el que aparece en los libros de texto, el que se imparte en nuestros centros de estudios, el que conocemos quienes hemos trabajado en mercadeo y ventas de grandes empresas, nacionales y multinacionales, de productos de consumo masivo, el que podemos apreciar cuando vamos a realizar nuestro mercado en las grandes superficies y cadenas de autoservicios, más aun si somos exigentes, no lo podemos llamar merchandising.

Las exhibiciones, espacios internos de ventas, actividades promocionales, etc., de los tenderos de barrio, las manejan de manera diferente, especial y compleja, según sus necesidades, realidades, vivencias, conocimientos, experiencias, creencias, mitos, religiones, amores, odios, miedos, su personalidad, entre la violencia y los desplazamientos, entre la informalidad $y$ la formalidad, entre sus incertidumbres, antagonismos, el orden y desorden, entre la luz y oscuridad, entre locales heterogéneos, que se autogeneran de manera recursiva, dialógicamente, etc.

$\mathrm{Si}$ le quisiéramos poner un nombre al merchandising encontrado lo podemos llamar 
como empírico y su otro componente sería el resultado de la emergencia social, (emergente), el primero fruto de su experiencia, de prueba y error. El segundo surge de su propia iniciativa y creatividad, de la relación con el barrio, sus compradores, clientes, proveedores, (quienes ejercen su mayor influencia y presión para lograr los mejores espacios que les permitan generar mayores ventas), competencia, de todos y cada uno de los factores descritos en el punto anterior, etc. d. Presentan muy altos grados de heterogeneidad. Las similitudes están en algunos muebles, enseres estandarizados. Como en las neveras, exhibidores, gancheras, etc., dados por los proveedores en comodato ${ }^{6}$.

Como ya se comentó aspectos como los mitos, la religión, creencias populares, están presentes en el ambiente, forma de atender a sus clientes, orientar sus negocios, etc.

En gran parte de las tiendas de barrio visitadas de la localidad estudiada, se pudo verificar, como muchos de los tenderos son "inmunes", a las estrategias y tácticas de las empresas proveedores, quienes invierten cuantiosas sumas de dinero, por ser este canal de distribución, el que mayor cantidad de ventas genera nivel agregado, según Fenaltiendas.

Muy relacionada con la anterior, el merchadising técnico o convencional, es decir elementos y acciones de mercadeo en sus tiendas prácticamente es escaso, mucho menos tácticas y estrategias preestablecidas. Si las emergentes.

Los espacios son muy reducidos, cuentan con escasos recursos económicos, muchas tiendas no fueron diseñadas para ello. La gran mayoría son casas de habitación, garajes, salas o comedores de sus lugares de habitación, fruto de adaptaciones locativas, muchas las cuales no

\footnotetext{
${ }^{6}$ Contrato por el cual se da o recibe prestada una cosa, en este caso un elemento o artefacto que facilita la venta de los productos de un proveedor o fabricante de productos de consumo masivo, de las que pueden usarse sin destruirse con la obligación de restituirla
}

cuentan con un diseño, decoración y ambiente adecuado.

A lo largo del presente estudio se demostró cómo tanto epistemológica, como empíricamente, es coherente y pertinente tratar las prácticas de merchandising de los tenderos de barrio, con miradas mercadológicas diferentes a las convencionales, que perfectamente se pueden constituir en un nuevo merchadising no tratado antes por autor alguno ${ }^{7}$. Que bajo enfoques lineales, positivistas $\mathrm{y}$ funcionales tratan el fenómeno de una manera superficial, reduccionista y determinista.

Como en el punto anterior el uso del diálogo y las narrativas como vehículo de investigación, demostró ser una adecuada técnica de recolección de información, en un segmento tan complejo y difícil de investigar, como son los tenderos de barrio, quienes no cuentan con tiempo disponible para responder las inquietudes de los investigadores.

\section{Referencias}

Anderson, R. (1977). "Schooling and the Acquisition of Knowledge. The notion of schemata and the educational enterprise". In R. C. Anderson, R. J. Spiro, \& W. E.

Bateson, G. (1972) "The Logical Categories of Learning and Communication. Steps to an

Ecology of Mind", The University of Chicago Press edition, pág. 279-308.

Díaz de Castro, E. (2006), "Merchandising, teoría y práctica”. Editorial Pirámide.España.

tkin y Schvarstein, (1995). "Identidad de las organizaciones. Invariancia y cambio", $3^{\text {a }}$. Ed.,

México D.F.Editorial Paidós.

Flores, F. (1994) "Creando Organizaciones para el Futuro". Santiago de Chile: Edit.Dolmen.

\footnotetext{
${ }^{7}$ De acuerdo con el rastreo efectuado en diversas bases de datos internacionales.
} 
Foucault, M.(1979). "La arqueología del saber”.6a . Ed., México D.F., Editorial Siglo XXI

Gergen K. (2007). "Construccionismo social, aportes para el debate y la práctica"; traductoras y compiladoras, Ángela María Estrada Mesa, Silvia Diazgranados Ferráns — Bogotá: Universidad de los Andes, Facultad de Ciencias Sociales, Departamento de Psicología, CESO, Ediciones Uniandes.

González, S. (2008), "Pensamiento complejo, Entorno a Edgar Morín, América Latina y los

Procesos Educativos", Colección Mesa Redonda, Cooperativa Editorial Magisterio, Bogotá, Colombia.

Kotler, P. (2009), "Dirección de Marketing". Editorial Prentice Hall, México.

Markova, I.(2000). "Amedée or how to get rid of it: social representations from a dialogical perspective, Papers on Social Representations", Noruega, Universidad de Wagenigen.
Maturana, H. y Varela H. (1999), “El árbol del conocimiento. Las bases biológicas del

Conocimiento humano", Madrid, España. Edit. Debate. S.A.

Morín, Edgar (1994): "Epistemología de la complejidad", en D. Fried Schnitman edtr. Nuevos paradigmas, cultura y subjetividad, Buenos Aires-Barcelona-México:

Paidós, Munné, F. (2005). “QQué es la complejidad?" Encuentros de Psicología Social,3 (2), 6-17.

Wittgenstein Ludwig. (1950) "Investigaciones Filosóficas", Madrid, España Alianza Editorial, S. A. 